\title{
Transcriptomic analysis of Lactococcus chungangensis sp. nov. and its potential in cheese making
}

\author{
Maytiya Konkit, ${ }^{*}$ Jong-Hwa Kim, ${ }^{*}$ Nagamani Bora, $†$ and Wonyong Kim ${ }^{* 1}$ \\ *Department of Microbiology, Chung-Ang University College of Medicine, Seoul, Republic of Korea 156-756 \\ †Department of Pharmacy, De Montfort University, Leicester, LE1 9BH, United Kingdom
}

\begin{abstract}
Lactococcus lactis has a played a prominent role in food industry from traditional milk fermentations to industrial scale processes. Extensive studies on the biochemical, physiological, and genetic aspects of $L$. lactis are evident from published literature. Recently, another novel species, Lactococcus chungangensis, was isolated from activated sludge as the sixth member of the genus to be discovered. To date, no study has been conducted to explore the functional aspects of $L$. chungangensis to identify features similar to those in L. lactis that are relevant to the dairy industry. Hence, the present study was undertaken to identify functional genes relevant to dairy application through comparative transcriptomic analysis of $L$. chungangensis with L. lactis. In expression microarray data, 415 genes were upregulated and 1,500 were downregulated of the total 1,915 probes analyzed. Interesting findings from this study were the identification of functional genes such as aminohydrolase and S-adenosylmethionine in L. chungangensis, which are useful in flavor production in cheese making. Probing these genes by PCR and analyzing the sequence confirmed the presence of these genes. Phenotypic analysis of these genes was also investigated by growing the strain in different concentrations of skim milk media to confirm the ability of L. chungangensis to degrade casein in milk, which is the major precursor for flavor enhancing compounds. Other adaptive and stress-response genes such as cold shock and heat shock proteins were also revealed. All experimental investigations at the functional level suggest that $L$. chungangensis possesses some interesting genes which are of commercial significance, especially in cheese production.
\end{abstract}

Key word: Lactococcus chungangensis, transcriptomics, MetC gene, CysK gene, cheese

\footnotetext{
Received April 28, 2014.

Accepted September 4, 2014.

${ }^{1}$ Corresponding author: kimwy@cau.ac.kr
}

\section{INTRODUCTION}

Dairy lactic acid bacteria (LAB) play an important role in dairy industry and are known for their generally recognized as safe status in producing healthy and flavorsome foods. Hence, LAB have been studied extensively to date to enhance both fundamental and application-based knowledge. Members of the Lactococcus genus are noted for their ability to produce the L-lactic acid enantiomer from glucose (Schleifer et al., 1985). The genus Lactococcus belongs to the family Streptococcaceae, and consists of 9 validly described Lactococcus species (http://www.bacterio.net/lactococcus. html). Lactococcus lactis is the type species of the genus, which has been routinely used in dairy industry in the production of cheese, yogurt, sour cream, and fermented milk (Cretenet et al., 2011). In cheese making, L. lactis ssp. lactis and L. lactis ssp. cremoris are used as starter cultures in several types of cheese such as cream cheese, Cheddar cheese, and Swiss cheese (Leroy and De Vuyst, 2004). Other species in the genus such as L. raffinolactis also play an important role in making Moroccan soft white cheese (Ouadghiri et al., 2005) and Egyptian Domiati cheese (El-Baradei et al., 2007).

Lactic acid bacteria degrade proteins and use several metabolic routes in producing flavor compounds while they thrive in milk. Thus, their metabolic end products and enzymes have significant influence in determining the texture and flavor of the final product (Samaržija, 2011; Kim, 2014). One of the main precursors for flavor compounds in milk is casein, a milk protein that is also derived from fatty acids and sugars (Liu et al., 2008). The proteolytic system of LAB degrades casein into different amino acid constituents such as methionine and cysteine, which are sulfur-containing amino acids. The volatile sulfur compounds such as methanethiol and hydrogen sulfide, the odor compounds in several types of cheese are the end products of metabolism of methionine and cysteine in dairy bacteria (Bogicevic et al., 2012). S-Adenosylmethionine synthase is one such enzyme which is involved in methionine catabo- 
lism, forming a precursor to methanethiol, one of the primary flavor compounds found in Cheddar cheese (Weimer et al., 1999) besides playing a role in other metabolic processes and general maintenance of cells. S-Adenosylmethionine is derived from the pathways of methionine and cysteine biosynthesis pathways in lactic acid bacteria (Irmler et al., 2009).

Lactococcus chungangensis CAU $28^{\mathrm{T}}$ was isolated from activated sludge and characterized against 5 recognized species by comparing phenotypic properties and cellular fatty acid composition, and by $16 \mathrm{~S}$ rRNA gene sequencing (Cho et al., 2008). Phenotypic characterization alone was not enough to delineate $L$. chungangensis CAU $28^{\mathrm{T}}$ from other Lactococcus species. The major fatty acid components were saturated fatty acids C16:0 and C14:0 and unsaturated fatty acid C18:1v7c; however, the fatty acid profile of this strain was similar to those of other reference Lactococcus type strains except Lactococcus piscium DSM 6634 ${ }^{\mathrm{T}}$. Genomic studies are the way forward to delineate this species and also to understand its unique characteristics. Until now, this strain has not been subject to genomic characterization, which has the potential to identify genes that are specific to this strain and also of commercial interest, especially in cheese making.

Whole-genome sequencing to elucidate the genetic content of a microorganism is considered to be an expensive and time-consuming procedure. Approaches that alleviate the necessity to sequence a large number of genes and that identify unique and common genes without sequencing are a trend in the present era. High-throughput microarrays are one such technology by which it is easier to identify both favorable and unfavorable genes. So far, gene expression microarrays are commonly used to determine the genomic content of bacteria, including inter-species comparison (Hakenbeck et al., 2001; Fukiya et al., 2004; van Hijum et al., 2008; Park et al., 2012). Gene expression microarray analysis is also widely used to answer important biological questions at a molecular level that are not easily answered using other detection techniques (dos Reis et al., 2003; Joo et al., 2011).

In the dairy industry, genome-wide comparisons of both starter and nonstarter LAB have been done, but no studies at the transcriptome level have been reported. A strategy was adopted in the current study to identify the functional genes of commercial significance without the need to sequence the whole genome. Gene expression analysis was done on $L$. chungangensis by in vitro transcriptome microarray experiments using an open reading frame microarray platform based on the L. lactis ssp. lactis I11403 platform.

\section{MATERIALS AND METHODS}

\section{Cultivation of Bacteria, RNA Extraction, and cDNA Synthesis and Labeling}

Lactococcus chungangensis $\mathrm{CAU} 28^{\mathrm{T}}$ was grown along with the reference strain L. lactis ssp. lactis KCTC $3769^{\mathrm{T}}$ on tryptic soy agar (TSA; Becton Dickinson, Sparks, MD) at $30^{\circ} \mathrm{C}$ for $18 \mathrm{~h}$. RNeasy Mini kit (Qiagen, Valencia, CA) was used to extract total RNA. The concentration of RNA was checked by Agilent 2100 Bioanalyzer (Agilent Technologies, Palo Alto, CA) and standardized. cDNA was synthesized from RNA by reverse transcription with the SuperScript doublestranded cDNA synthesis kit using an oligo dT primer from cDNA synthesis kit (Invitrogen Life Technologies, Carlsbad, CA). Briefly, $10 \mu \mathrm{g}$ of total RNA was reverse-transcribed to cDNA using an oligo dT primer, then second-strand cDNA synthesis was performed. After purification, the cDNA was quantified using the ND-1000 spectrophotometer (NanoDrop, Wilmington, DE). The cDNA was labeled using the One-Color Labeling Kit (Nimblegen, Madison, WI) according to the Nimblegen expression protocol. One microgram of each cDNA sample was labeled with Cy3 using Cy3-random nonamer. After purification, the labeled cDNA was quantified using the NanoDrop ND-1000 spectrophotometer.

\section{Microarrays, Data Acquisition, and Statistical Analysis}

The custom microarray L. lactis pangenome $385 \mathrm{~K}$ short oligo array (Nimblegen), with 2,321 genes: 735,308 probes, and 5 replicates, was mapped onto the wholegenome data from L. lactis ssp. lactis Il1403 (GenBank accession number NC_002662.1) and whole-genome scaffolds for L. chungangensis (unpublished data). The free energy of hybridization, Go, of probes to cDNA was calculated using the HyTherTM (SantaLucia, 1998; SantaLucia and Hicks, 2004) web service (http:// ozone3.chem.wayne.edu/cgi-bin/login/login/login Verify.cgi) in $1 \times \mathrm{SSC}$ at $42^{\circ} \mathrm{C}$ with the default concentrations, and the correction for microchips was applied (Fotin et al., 1998). Probes with calculated Go $<-45$ $\mathrm{kcal} / \mathrm{mol}$ were treated as giving a full hybridization signal, whereas probes of the draft genome of L. chungangensis with Go $>-25 \mathrm{kcal} / \mathrm{mol}$ were treated as failing to hybridize. Signals from probes with a free energy of hybridization between -25 and $-45 \mathrm{kcal} / \mathrm{mol}$ were corrected by $15 \%$ per kcal/mol. NimbleScan (version 2.5, Gene Expression RMA algotithm) was used to extract raw data and logarithmic transformation (base2) of the 
signals was performed at $532 \mathrm{~nm}$ with a resolution of $2 \mu \mathrm{m}$. The signals on the L. chungangensis CAU $28^{\mathrm{T}}$ were normalized to the L. lactis, based upon the signal levels of a set of structural ribosomal proteins, by 0.2 $x$ the corrected signal levels. An increase in normalized signal level of at least 2-fold was used to determine upregulated gene expression. Hierarchical cluster analysis was performed using complete linkage and Euclidean distance as a measure of similarity, as shown in Park et al. (2012). The analyzed data has been deposited in NCBI's Gene Expression Omnibus (Edgar et al., 2002) and is accessible through GEO series accession number GSE58002 (http://www.ncbi.nlm.nih.gov/geo/query/ acc.cgi? acc $=$ GSE58002).

\section{PCR Amplification of MetC and CysK Genes}

Genomic DNA from L. chungangensis CAU $28^{\mathrm{T}}$ and L. lactis ssp. lactis KCTC $3769^{\mathrm{T}}$ were extracted by i-genomic BYF DNA Extraction mini kit (iNtRON Biotechnology, Seoul, Korea). Primer sets were designed targeting methionine and cysteine biosynthesis including the S-adenosylmethionine synthase pathway involved in sulfur metabolism (Fernandez et al., 2002). Primer sets were designed for cystathionine $\beta$-lyase $(\mathrm{Met} C)$ gene (5'-CTGGAATTCATGCGGTTCTT-3' and 5'-ACGCCACCTAGTGATTCAGC-3') and O-acetylserine sulfhydrylase (CysK) gene (5'-ACAGAAGTGCATACAATTCA-3' and 5'-GATTAAGAAGATTAAAGGAGG-3') using PRIMER3 software (Rozen and Skaletsky, 1999) based on the gene sequence of L. lactis ssp. cremoris MG1363. A PCR reaction mixture (20 $\mu \mathrm{L}$ ) consisting of $5 \mathrm{U}$ of Taq DNA polymerase (BEAMS Biotechnology, Seongnam, Korea), $2.0 \mu \mathrm{L}$ of $10 \times$ Taq buffer, $1 \mu \mathrm{L}$ of dNTP mixture, $1 \mu \mathrm{L}$ at $10 \mathrm{~m} M$ of each primer, and $3 \mu \mathrm{L}$ of genomic DNA template was used for amplification of target genes. Amplifications were performed in a TProfessional Thermocycler (Biometra GmbH, Göttingen, Germany) with an initial denaturation step at $95^{\circ} \mathrm{C}$ for $5 \mathrm{~min}, 30$ repeated cycles of $95^{\circ} \mathrm{C}$ for $1 \mathrm{~min}$, annealing $55^{\circ} \mathrm{C}$ for $1 \mathrm{~min}$ and extension at $72^{\circ} \mathrm{C}$ for $1 \mathrm{~min}$, and a final cycle of extension at $72^{\circ} \mathrm{C}$ for $10 \mathrm{~min}$. Three microliters of amplified PCR products were electrophoresed on $1.2 \%$ Seakem LE agarose gel (FMC Bioproducts, Rockland, ME) and the gel was visualized with Gel DOC $\mathrm{XR}^{+}$Imaging system (Bio-Rad Laboratories, Hercules, CA). The PCR products were sequenced by using a BigDye Terminator Cycle Sequencing kit and an automatic DNA sequencer (model 3730; Applied Biosystems, Foster City, CA). Sequences were aligned using the CLUSTAL_X 1.81 program (Thompson et al., 1997) with parameters set against corresponding sequences from the NCBI GenBank database. The nucleotide sequences obtained in this study were deposited in GenBank under the accession numbers KM073976 (methionine) and KM073977 (cysteine), respectively.

\section{Growth Conditions in Different Concentrations of Skim Milk Media}

The coagulation and proteolysis of casein in milk was tested by adding skim milk powder (Becton Dickinson) to TSA (Becton Dickinson). To compare the proteolytic ability of $L$. chungangensis $\mathrm{CAU} 28^{\mathrm{T}}$, the reference strain L. lactis ssp. lactis $\mathrm{KCTC} 3769^{\mathrm{T}}$ was grown in the same media and incubated at $30^{\circ} \mathrm{C}$ for 48 h. The growth pattern of $L$. chungangensis CAU $28^{\mathrm{T}}$ was observed in $5 \%$ and $20 \%$ liquid skim milk media cultivated aerobically at $30^{\circ} \mathrm{C}$ in triplicates with a control strain. Cell densities were measured using Infinite 200 PRO NanoQuant (Tecan, Männedorf, Switzerland) spectrophotometer at a wavelength of $600 \mathrm{~nm}$.

\section{RESULTS}

\section{Microarray Statistical Analysis}

The comparison of the expression profiles by hybridization to the immobilized probes on the microarray of L. lactis ssp. lactis Il1403 (GenBank accession number NC002662) with the total RNA of L. chungangensis CAU $28^{\mathrm{T}}$, showed reproducibility with interarray correlation values (range: $-1 \leq \mathrm{r} \leq 1$ ) and pairwise scatter plots of gene expression values $\left(\log _{2}\right)$ as shown in Figure 1. A correlation value (r) of 0.29 was found between reference strain $L$. lactis and the tester strain $L$. chungangensis, suggesting a positive correlation between the two. Of the 1,915 genes analyzed, the expression level was upregulated for 415 genes and downregulated for 1,500 genes in L. chungangensis. The Euclidean hierarchical clustering method with normalized signal shows upregulated genes in red and downregulated genes in green (Figure 2).

\section{Identification of Upregulated and Downregulated Genes in $L$. chungangensis}

Genes that were upregulated and downregulated were identified, especially those involved in flavor formation in cheese making using a cluster of orthologous groups (COG) approach (ftp://ftp.ncbi.nih.gov/pub/ wolf/COGs/COG0303/fun.txt). The majority of upand downregulated genes belonged to translation and transcription ( $\mathrm{J}$ and $\mathrm{K}$ ), DNA replication $(\mathrm{L})$, cellular division and cell envelope (D and $\mathrm{M}$ ), posttranslational modification $(\mathrm{O})$, energy production $(\mathrm{C})$, carbohydrate transport and metabolism $(\mathrm{G})$, amino acid transport 


\section{L. chungangensis $\mathrm{CAU} \mathbf{2 8}^{\mathrm{T}}$}

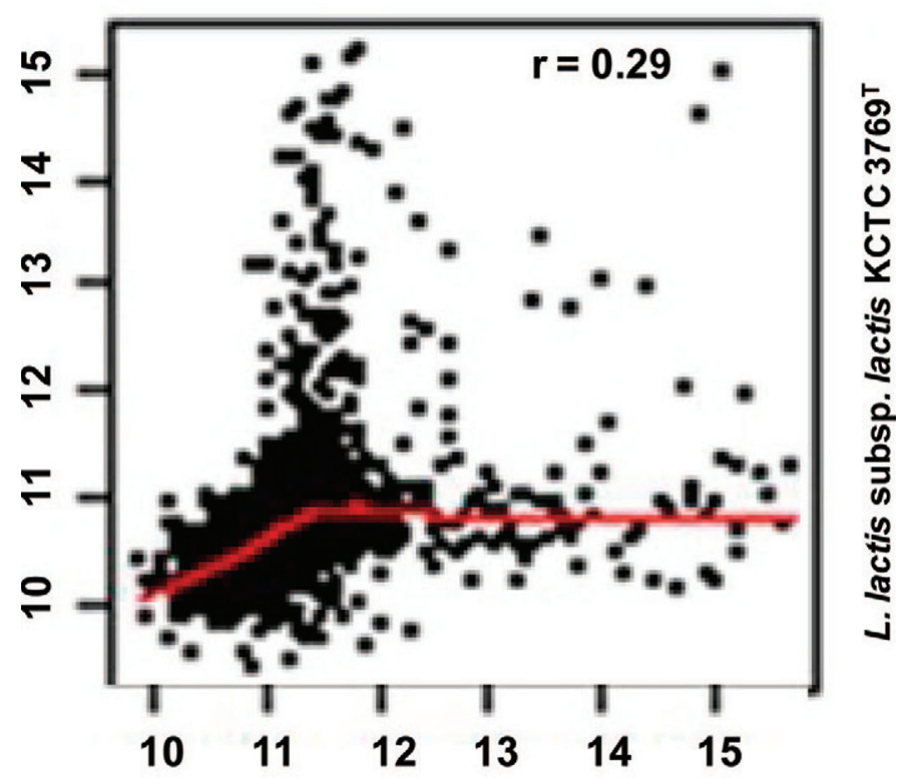

Normalized value

Figure 1. Reproducibility and dynamic range with pairwise scatter plot. Gene expression values are shown in terms of $\log _{2}$. Color version available in the online PDF.

and metabolism $(\mathrm{E})$, inorganic ion transport $(\mathrm{P})$, and function unknown $(\mathrm{S})$ categories (Table 1). The 39 examples out of 415 upregulated genes that were classified as related to cheese flavor production included amino acid amidohydrolase, S-adenosylmethionine synthase, alanine racemase, and glutamate decarboxylase, which were classified in amino acid transport and metabolism (E), and glyceraldehyde-3-phosphate dehydrogenase, L-lactate dehydrogenase, and mannitol-specific PTS system IIA component, which were classified in carbohydrate transport and metabolism (G). Interestingly, stress-response genes which were classified in transcription (A) and posttranslational modification, protein turnover, chaperones $(\mathrm{O})$, such as cold shock protein E, a 2-component system regulator, and molecular chaperone DnaK, were also upregulated.

Notable findings from this study were the identification of functional genes such as aminohydrolase and S-adenosylmethionine that were upregulated in $L$. chungangensis which play a role in flavor production, especially S-adenosylmethionine, related to flavor production in cheese through the methionine and cysteine pathway.

The findings also identified 1,500 downregulated genes in L. chungangensis CAU $28^{\mathrm{T}}$. Thirty-nine examples of downregulated genes were also classified using the cluster of orthologous groups approach (Table
1). Genes were classified in amino acid transport and metabolism (E), such as peptide binding protein and intercellular adhesion protein. Ferrous ion transport protein A, multidrug efflux transporters, and cationic amino acid transport were classified in inorganic ion transport and metabolism $(\mathrm{P})$. Genes were also classified in carbohydrate transport and metabolism (E), such as $\beta$-glucosidase and phospho- $\beta$-glucosidase. Stress-response genes such as NADH oxidases were classified in energy production and conversion (C). The 39 examples of up- and downregulated genes shown in Table 1 were related with hierarchical mapping (Figure 2) showing the relative level of expression; red represents high expression.

\section{Sequencing Results of Cheese-Making Flavor Genes}

The cystathionine $\beta$-lyase $(\mathrm{Met} C)$ and $\mathrm{O}$-acetylserine sulfhydrylase $(C y s K)$ genes are important factors in determining the flavor-enhancing aspects in L. chungangensis. Sequencing the MetC and CysK genes of the L. chungangensis CAU $28^{\mathrm{T}}$ strain shows that $M e t C$ is $684 \mathrm{bp}$ and $C y s K$ is $927 \mathrm{bp}$ in length. The sequence identities of the MetC and CysK genes of the L. chungangensis $\mathrm{CAU} 28^{\mathrm{T}}$ strain were 63.7 to $66.6 \%$ for $L$. lactis ssp. lactis and 69.3 to $66.8 \%$ for L. lactis ssp. cremoris.

\section{Phenotypic Analysis of Functional Genes in L. chungangensis and Their Application in Cheese Making}

Phenotypic analysis of the coagulation and proteolysis of casein in L. chungangensis $\mathrm{CAU} 28^{\mathrm{T}}$ was investigated. The strain can grow on skim milk media up to $20 \%$ as indicated by the clear zone on the TSAskim milk agar plates (Figure 3). Proteolytic bacteria can hydrolyze casein to form soluble nitrogenous compounds, as indicated by the clear zone surrounding the colonies. The growth pattern in both $5 \%$ and $20 \%$ skim milk media showed no exponential growth, although higher cell densities were noted in $20 \%$ skim milk media (Figure 4). No significant difference was found in the growth patterns of $L$. chungangensis CAU $28^{\mathrm{T}}$ and $L$. lactis ssp. lactis KCTC $3769^{\mathrm{T}}$ in skim milk media.

\section{DISCUSSION}

Lactic acid bacteria play an essential role in the food industry with their notable metabolism, contributing to the sensory characteristics of the products and inhibiting the growth of undesirable microorganisms, ensuring the quality and safety of the final product. Extensive research has been done on this group of microbes, which 


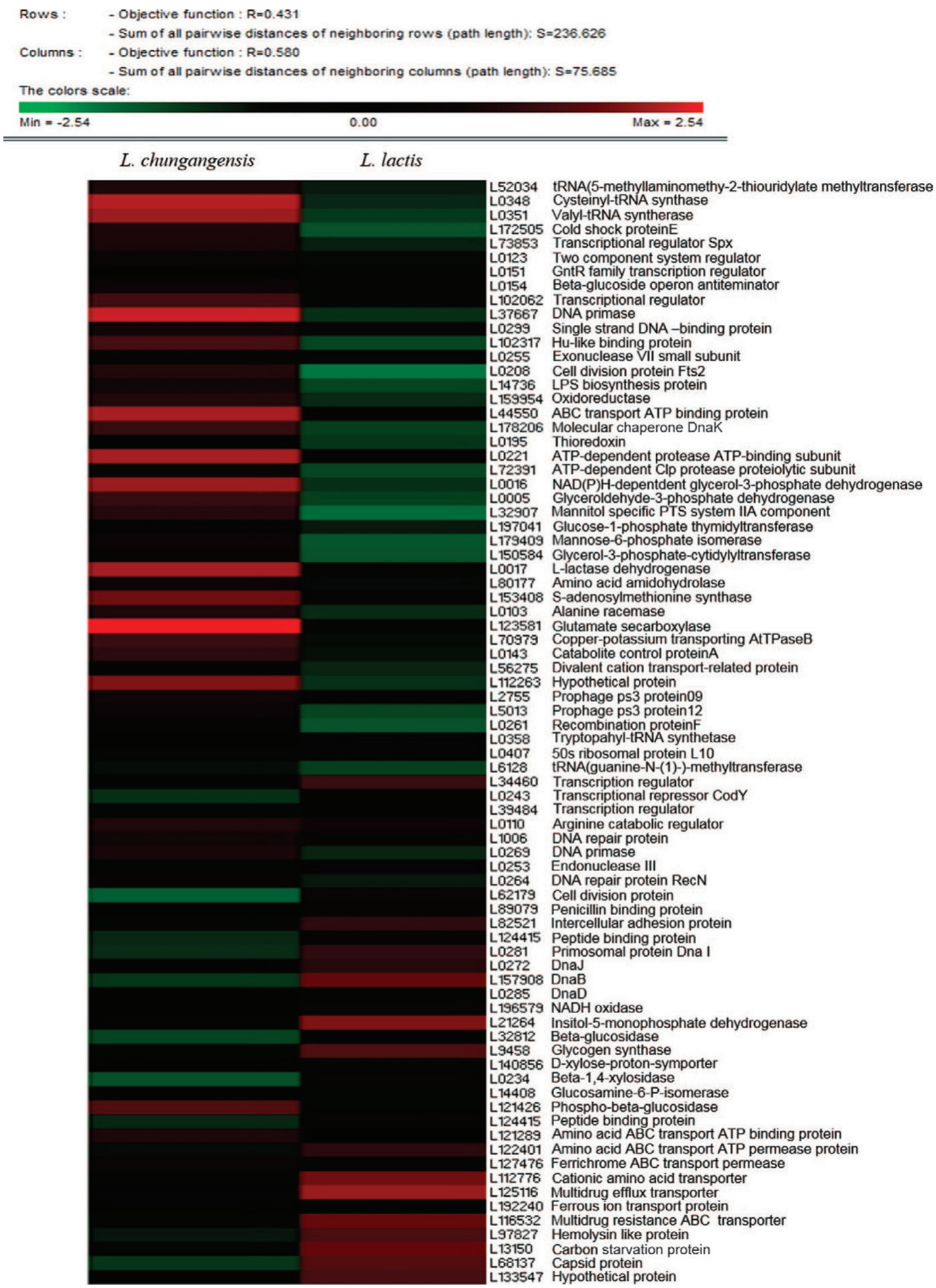

Figure 2. Hierarchical map of differentially expressed genes in cheese-making flavor functions. Black (red) represents high expression; gray (green) represents low expression. Color version available in the online PDF.

includes isolation of novel strains which are adaptable, robust, and have superior qualities to existing strains. The current study is aimed at identifying such novel properties with the potential to enhance the quality of the product in cheese making
Comparative transcriptomics was performed on this strain with the reference strain $L$. lactis ssp. lactis KCTC $3769^{\mathrm{T}}$. Global transcriptomic analysis of both strains shows a low relationship of $L$. chungangensis CAU $28^{\mathrm{T}}$ with L. lactis ssp. lactis KCTC $3769^{\mathrm{T}}$. This low 
Table 1. List of 39 upregulated and downregulated genes involved in cheese-making flavor in L. chungangensis CAU $28^{\mathrm{T}}$ using a cluster of orthologous group approach

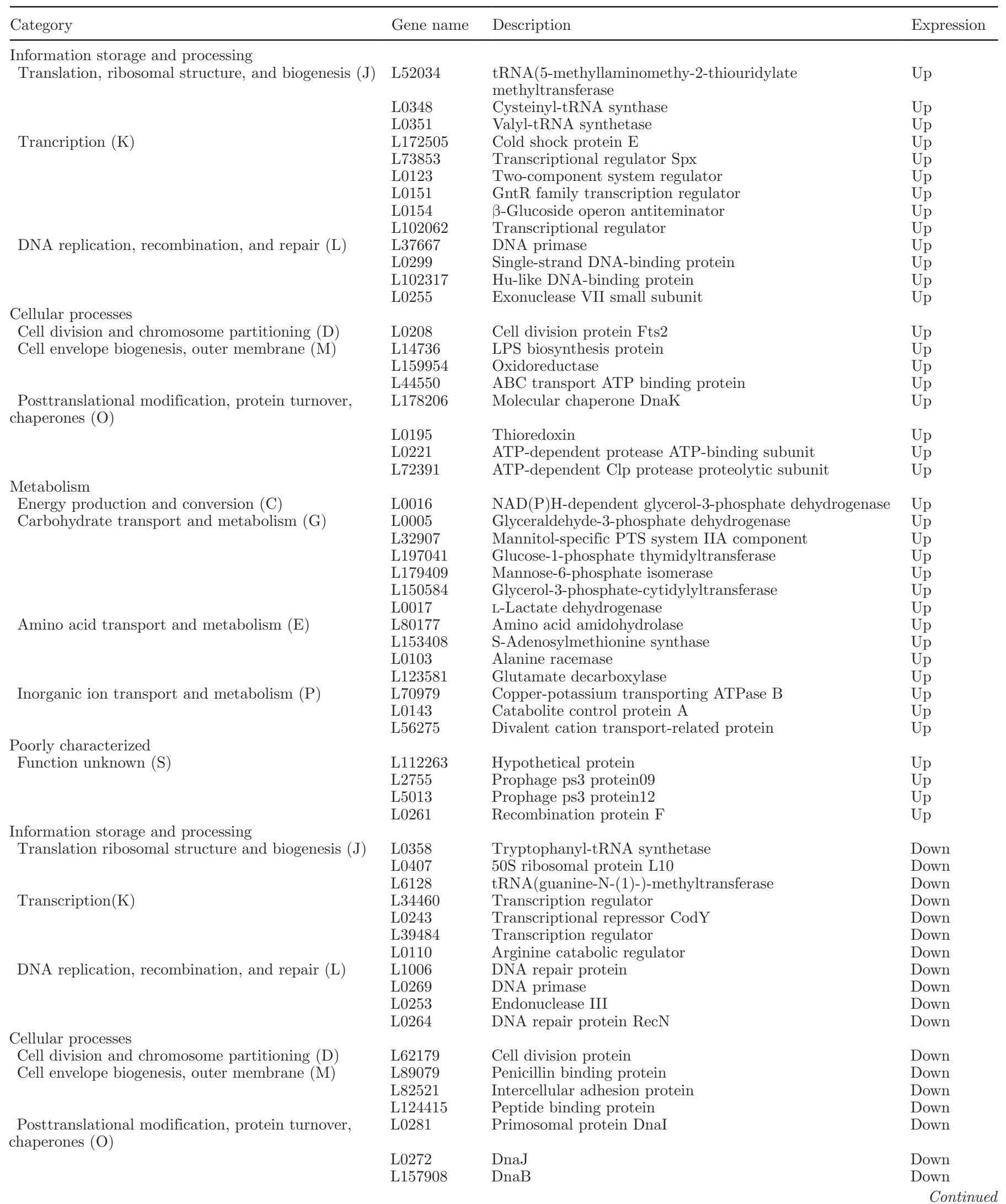


Table 1 (Continued). List of 39 upregulated and downregulated genes involved in cheese-making flavor in L. chungangensis CAU $28^{\mathrm{T}}$ using a cluster of orthologous group approach

\begin{tabular}{|c|c|c|c|}
\hline Category & Gene name & Description & Expression \\
\hline \multicolumn{4}{|l|}{ Metabolism } \\
\hline Energy production and conversion $(\mathrm{C})$ & L196579 & NADH oxidase & Down \\
\hline \multirow[t]{3}{*}{ Carbohydrate transport and metabolism (G) } & L3281 & $\beta$-Glucosidase & Down \\
\hline & L9458 & Glycogen synthase & Down \\
\hline & L140856 & D-xylose proton-symporter & Down \\
\hline \multirow[t]{4}{*}{ Amino acid transport and metabolism (E) } & L124415 & Peptide binding protein & Down \\
\hline & L121289 & Amino acid $\mathrm{ABC}$ transport ATP binding protein & Down \\
\hline & L122401 & Amino acid $\mathrm{ABC}$ transport ATP permease protein & Down \\
\hline & L127476 & Ferrichrome ABC transport permease & Down \\
\hline \multirow[t]{2}{*}{ Inorganic ion transport and metabolism $(\mathrm{P})$} & L11277 & Cationic amino acid transporter & Down \\
\hline & L125116 & Multidrug efflux transporter & Down \\
\hline & L68137 & Capsid protein & Down \\
\hline & L133547 & Hypothetical protein & Down \\
\hline
\end{tabular}

correlation was observed at least because of sequence divergence of $L$. chungangensis $\mathrm{CAU} 28^{\mathrm{T}}$ from L. lactis ssp. lactis KCTC $3769^{\mathrm{T}}$. The multiple probes per gene on the Nimblegen platform mean that probes to more conserved regions in the genes still hybridize success- fully. Of the 415 upregulated genes in L. chungangensis CAU $28^{\mathrm{T}}$, functions relating to protein metabolism, such as amino acid aminohydrolase, alanine racemase, and glutamate decarboxylase, are noteworthy. These genes are a part of the proteolytic system in LAB and
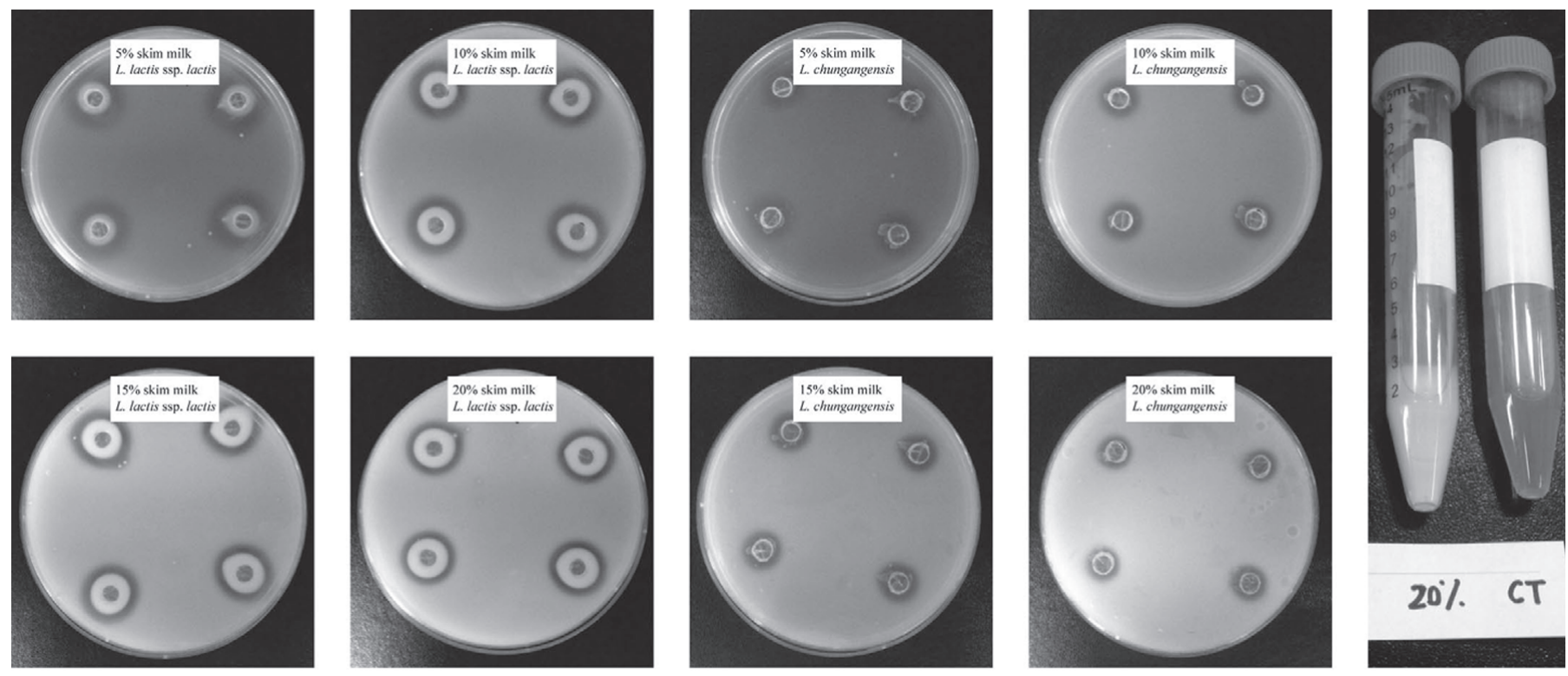

(a)

(b)

(c)

Figure 3. (a) The growth of Lactococcus lactis ssp. lactis KCTC $3769^{\mathrm{T}}$ and (b) Lactococcus chungangensis CAU $28^{\mathrm{T}}$ on tryptic soy agar with $5 \%, 10 \%, 15 \%$, and $20 \%$ skim milk agar plates, and (c) coagulation of $L$. chungangensis CAU $28^{\mathrm{T}}$ in $20 \%$ skim milk media in comparison with a noncoagulating control strain (CT). 


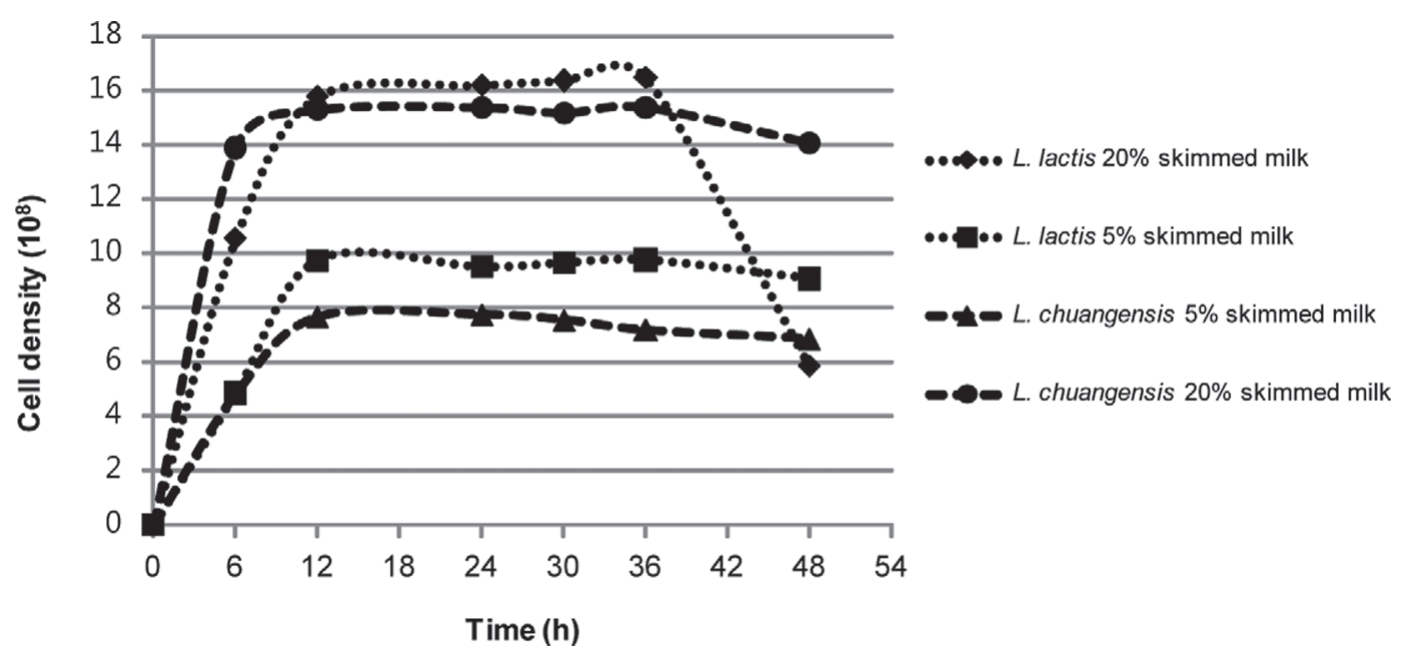

Figure 4. Physiological growth pattern of Lactococcus chungangensis CAU $28^{\mathrm{T}}$ and Lactococcus lactis ssp. lactis $3769^{\mathrm{T}}$ in skim milk media.

are involved in biosynthesis and catabolism of proteins into amino acids and peptides, and have proven to be crucial for cheese ripening.

In cheese making, strains that have the capacity to grow up to high cell densities producing lactic acid and to ferment milk carbohydrates are of commercial importance. Milk is a very complex medium that changes continuously during fermentation. It consists of the milk protein casein that is degraded by the bacterial proteases into peptides and amino acids, as well as vitamins (de Jong et al., 2013). At high cell densities, amino acids in milk may not suffice for the entire growing population of cells. Therefore, the alternative is to resort to extracellular hydrolysis of large proteins such as casein into short peptides and amino acids before transport for use as flavor precursors in cheese production (Xie et al., 2004).

Lactococci have such a proteolytic system, which is the key for flavor formation in cheese (Urbach, 1995). The hydrolyzed amino acids also act as precursors for a multitude of volatile compounds. The growth of L. chungangensis $\mathrm{CAU} 28^{\mathrm{T}}$ in $20 \%$ skim milk media demonstrates that this organism can degrade casein in milk and produce acid from fermentable carbohydrates, proving it to be useful in proteolysis of cheese ripening, contributing to the texture and flavor of cheese. Higher cell densities were recorded in $20 \%$ compared with 5\% skim milk media, supporting the presence of enzymes necessary for hydrolysis of proteins. One of the functional genes expressed in L. chungangensis CAU $28^{\mathrm{T}}$ is S-adenosylmethionine synthase, which is involved in methionine catabolism. It is a precursor to methanethiol, one of the primary flavor compounds found in Cheddar cheese (Weimer et al., 1999) besides playing a role in other metabolic processes and general maintenance of the cell.
Furthermore, identification of $M e t C$ and $C y s K$ genes supports the flavor-enhancing capacity of L. chungangensis CAU $28^{\mathrm{T}}$, as both genes are a part of cysteine and methionine metabolism pathways that lead to the formation of volatile sulfur compounds such as methanethiol, which is the dominant odor-active compound in many varieties of cheese (Bogicevic et al., 2012).

Compared with gene sequences with $L$. lactis ssp. lactis and L. lactis ssp. cremoris, which play important roles in the processing of cheese (Leroy and De Vuyst, 2004), the identities among species were over $60 \%$ in both of $M e t C$ and $C y s K$ genes. Generally, it is known that it is difficult to find the differences at the interspecies level of microorganisms using cDNA microarray hybridization (Aguado-Urda et al., 2010). However, our finding is interesting in that transcriptomic microarray analysis could successfully identify functional genes without laborious whole-genome sequencing analysis for industrial purposes.

In the present study, upregulated genes relating to carbohydrate and sugar metabolism, such as multiple sugar $\mathrm{ABC}$ transporters are common to most bacteria, not only LAB. These transporters might have specificity to oligosaccharides such as raffinose and are known to transport multiple substrates.(Ajdic and Pham, 2007).

As revealed by transcriptomic analysis, L. chungangensis also harbors stress-response genes such as cold shock protein E, superoxide dismutase, and 2-component system regulator. Such stress-response genes are useful in cheese making, as strains are subjected to acid, cold, and heat stress (Xie et al., 2004). For example, cold shock protein E prevents protein misfolding during cold stress, whereas superoxide dismutase activity helps combat acid stress, as revealed by previous studies in acid stress-induced protein expression in L. lactis (Sanders et al., 1995). Similarly, heat shock 
proteins are involved in the maturation of newly synthesized proteins, proper refolding, or degradation of denatured protein (Georgopoulos and Welch, 1993; Hartl et al., 1994). Most organisms respond to heat shock by synthesizing a conserved set of proteins, such as the DnaK-DnaJ-GrpE and GroEL-GroES chaperone complex (Yura et al., 1993). In contrast, the NADH oxidase common oxidative stress resistance mechanism found in L. lactis (Miyoshi et al., 2003) was absent in L. chungangensis.

In comparison with $L$. lactis, some undesirable genes were downregulated in L. chungangensis CAU $28^{\mathrm{T}}$, such as multidrug efflux transporter and multidrug resistance $\mathrm{ABC}$ transporter, which usually play a major role in intrinsic resistance in gram-negative bacteria (Levy, 1992). The latter are considered to form pores in the cytoplasmic membrane of erythrocytes, leukocytes, and other cells, leading to modification of cellular functions, lysis of host cells, or both (Sakiyama et al., 2006). The absence of such genes in L. chungangensis makes it more desirable for food fermentation.

Functional genes involved in degradation of casein, tolerance to stress conditions, and ability to use a variety of substrates were revealed by transcriptomic analysis of $L$. chungangensis $\mathrm{CAU} 28^{\mathrm{T}}$, proving it to be useful as a starter or nonstarter strain in cheese manufacturing. Further investigation on the ability of the strain to inhibit the growth of other food spoilage organisms should make it economically more viable. This pilot study should lead to adaptation of the strain, probing the physiology further for fermentation at laboratory and industrial scales.

\section{REFERENCES}

Aguado-Urda, M., G. H. Lopez-Campos, J. F. Fernandez-Garayzabal, F. Martin-Sanchez, A. Gibello, L. Dominguez, and M. M. Blanco. 2010. Analysis of the genome content of Lactococcus garvieae by genomic interspecies microarray hybridization. BMC Microbiol. 10:79.

Ajdic, D., and V. T. Pham. 2007. Global transcriptional analysis of Streptococcus mutans sugar transporters using microarrays. J. Bacteriol. 189:5049-5059.

Bogicevic, B., H. Berthoud, R. Portmann, L. Meile, and S. Irmler. 2012. CysK from Lactobacillus casei encodes a protein with O-acetylserine sulfhydrylase and cysteine desulfurization activity. Appl. Microbiol. Biotechnol. 94:1209-1220.

Cho, S. L., S. W. Nam, J. H. Yoon, J. S. Lee, A. Sukhoom, and W. Kim. 2008. Lactococcus chungangensis sp. nov., a lactic acid bacterium isolated from activated sludge foam. Int. J. Syst. Evol. Microbiol. 58:1844-1849.

Cretenet, M., V. Laroute, V. Ulvé, S. Jeanson, S. Nouaille, S. Even, M. Piot, L. Girbal, Y. Le Loir, and P. Loubière. 2011. Dynamic analysis of the Lactococcus lactis transcriptome in cheeses made from milk concentrated by ultrafiltration reveals multiple strategies of adaptation to stresses. Appl. Environ. Microbiol. 77:247-257.

de Jong, A., M. E. Hansen, O. P. Kuipers, M. Kilstrup, and J. Kok. 2013. The transcriptional and gene regulatory network of Lactococcus lactis MG1363 during growth in milk. PLoS ONE 8:e53085.

dos Reis, M., L. Wernisch, and R. Savva. 2003. Unexpected correlations between gene expression and codon usage bias from micro- array data for the whole Escherichia coli K-12 genome. Nucleic Acids Res. 31:6976-6985.

Edgar, R., M. Domrachev, and A. E. Lash. 2002. Gene expression omnibus: NCBI gene expression and hybridization array data repository. Nucleic Acids Res. 30:207-210.

El-Baradei, G., A. Delacroix-Buchet, and J.-C. Ogier. 2007. Biodiversity of bacterial ecosystems in traditional Egyptian Domiati cheese. Appl. Environ. Microbiol. 73:1248-1255.

Fernandez, M., M. Kleerebezem, O. P. Kuipers, R. J. Siezen, and R. van Kranenburg. 2002. Regulation of the metC-cysK operon, involved in sulfur metabolism in Lactococcus lactis. J. Bacteriol. 184:82-90.

Fotin, A. V., A. L. Drobyshev, D. Y. Proudnikov, A. N. Perov, and A. D. Mirzabekov. 1998. Parallel thermodynamic analysis of duplexes on oligodeoxyribonucleotide microchips. Nucleic Acids Res. 26:1515-1521.

Fukiya, S., H. Mizoguchi, T. Tobe, and H. Mori. 2004. Extensive genomic diversity in pathogenic Escherichia coli and Shigella strains revealed by comparative genomic hybridization microarray. J. Bacteriol. 186:3911-3921.

Georgopoulos, C., and W. J. Welch. 1993. Role of the major heat shock proteins as molecular chaperones. Annu. Rev. Cell Biol. 9:601-634

Hakenbeck, R., N. Balmelle, B. Weber, C. Gardes, W. Keck, and A. de Saizieu. 2001. Mosaic genes and mosaic chromosomes: intraand interspecies genomic variation of Streptococcus pneumoniae. Infect. Immun. 69:2477-2486.

Hartl, F. U., R. Hlodan, and T. Langer. 1994. Molecular chaperones in protein folding: The art of avoiding sticky situations. Trends Biochem. Sci. 19:20-25.

Irmler, S., H. Schäfer, B. Beisert, D. Rauhut, and H. Berthoud. 2009. Identification and characterization of a strain-dependent cystathionine $\beta / \gamma$-lyase in Lactobacillus casei potentially involved in cysteine biosynthesis. FEMS Microbiol. Lett. 295:67-76.

Joo, J. I., T. S. Oh, D. H. Kim, D. K. Choi, X. Wang, J. W. Choi, and J. W. Yun. 2011. Differential expression of adipose tissue proteins between obesity-susceptible and -resistant rats fed a high-fat diet. Proteomics 11:1429-1448.

Kim, W. 2014. The genus Lactococcus. Pages 429-443 in Lactic Acid Bacteria: Biodiversity and Taxonomy. W. H. Holzapfel and B. J. B. Wood, ed. Wiley Blackwell, Oxford, UK.

Leroy, F., and L. De Vuyst. 2004. Lactic acid bacteria as functional starter cultures for the food fermentation industry. Trends Food Sci. Technol. 15:67-78.

Levy, S. B. 1992. Active efflux mechanisms for antimicrobial resistance. Antimicrob. Agents Chemother. 36:695-703.

Liu, M., A. Nauta, C. Francke, and R. J. Siezen. 2008. Comparative genomics of enzymes in flavor-forming pathways from amino acids in lactic acid bacteria. Appl. Environ. Microbiol. 74:4590-4600.

Miyoshi, A., T. Rochat, J. J. Gratadoux, Y. Le Loir, S. C. Oliveira, P. Langella, and V. Azevedo. 2003. Oxidative stress in Lactococcus lactis. Genet. Mol. Res. 2:348-359.

Ouadghiri, M., M. Amar, M. Vancanneyt, and J. Swings. 2005. Biodiversity of lactic acid bacteria in Moroccan soft white cheese (Jben). FEMS Microbiol. Lett. 251:267-271.

Park, H. K., S. C. Myung, and W. Kim. 2012. Comparative transcriptomic analysis of Streptococcus pseudopneumoniae with viridans group streptococci. BMC Microbiol. 12:77.

Rozen, S., and H. Skaletsky. 1999. Primer3 on the WWW for general users and for biologist programmers. Pages 365-386 in Bioinformatics Methods and Protocols. Vol. 132 in Methods in Molecular Biology. Springer/Humana Press Inc., Totowa, NJ.

Sakiyama, T., H. Ueno, H. Homma, O. Numata, and T. Kuwabara 2006. Purification and characterization of a hemolysin-like protein, Sll1951, a nontoxic member of the RTX protein family from the Cyanobacterium Synechocystis sp. strain PCC 6803. J. Bacteriol. 188:3535-3542.

Samaržija, D., N. Antunac, and J. L. Havranek. 2011. Taxonomy, physiology and growth of Lactococcus lactis: A review. Mljekarstvo 51:35-48. 
Sanders, J. W., K. J. Leenhouts, A. J. Haandrikman, G. Venema, and J. Kok. 1995. Stress response in Lactococcus lactis: Cloning, expression analysis, and mutation of the lactococcal superoxide dismutase gene. J. Bacteriol. 177:5254-5260.

SantaLucia, J. Jr. 1998. A unified view of polymer, dumbbell, and oligonucleotide DNA nearest-neighbor thermodynamics. Proc. Natl. Acad. Sci. USA 95:1460-1465.

SantaLucia, J. Jr., and D. Hicks. 2004. The thermodynamics of DNA structural motifs. Annu. Rev. Biophys. Biomol. Struct. 33:415440

Schleifer, K. H., J. Kraus, C. Dvorak, R. Kilpperbalz, M. D. Collins, and W. Fischer. 1985. Transfer of streptococcus-lactis and related streptococci to the genus Lactococcus gen-nov. Syst. Appl. Microbiol. 6:183-195.

Thompson, J. D., T. J. Gibson, F. Plewniak, F. Jeanmougin, and D. G. Higgins. 1997. The CLUSTAL_X windows interface: Flexible strategies for multiple sequence alignment aided by quality analysis tools. Nucleic Acids Res. 25:4876-4882.
Urbach, G. 1995. Contribution of lactic acid bacteria to flavour compound formation in dairy products. Int. Dairy J. 5:877-903.

van Hijum, S. A., R. J. Baerends, A. L. Zomer, H. A. Karsens, V. Martin-Requena, O. Trelles, J. Kok, and O. P. Kuipers. 2008. Supervised Lowess normalization of comparative genome hybridization data-Application to lactococcal strain comparisons. BMC Bioinformatics 9:93.

Weimer, B., K. Seefeldt, and B. Dias. 1999. Sulfur metabolism in bacteria associated with cheese. Antonie van Leeuwenhoek 76:247261.

Xie, Y., L. S. Chou, A. Cutler, and B. Weimer. 2004. DNA macroarray profiling of Lactococcus lactis ssp. lactis IL1403 gene expression during environmental stresses. Appl. Environ. Microbiol. 70:6738-6747.

Yura, T., H. Nagai, and H. Mori. 1993. Regulation of the heat-shock response in bacteria. Annu. Rev. Microbiol. 47:321-350. 